\title{
Spontaneous infarction of pleomorphic adenoma of the parotid gland: A case report
}

\author{
HIROKO ITO $^{1}$, MITSUAKI ISHIDA ${ }^{1}$, KIMIAKI OKANO ${ }^{1}$, KAORI SANDOH $^{1}$, YUSUKE EBISU $^{1}$, \\ SAYA YOSHIOKA $^{1}$, TAKUO FUJISAWA ${ }^{1}$, HIROSHI IWAI ${ }^{2}$ and KOJI TSUTA ${ }^{1}$ \\ ${ }^{1}$ Department of Pathology and Laboratory Medicine and ${ }^{2}$ Department of Otolaryngology, \\ Kansai Medical University, Hirakata, 573-1010 Osaka, Japan
}

Received April 5, 2020; Accepted August 21, 2020

DOI: $10.3892 / \mathrm{mco} .2020 .2138$

\begin{abstract}
Infarction after the fine-needle aspiration (FNA) procedure is a well-recognized phenomenon of salivary gland lesions. Though extremely rare, spontaneous infarction without previous FNA can occur in pleomorphic adenoma (PA). The present report describes a fourth cytological case of spontaneous infarction of PA of the parotid gland using immunocytochemical analysis, to the best of our knowledge, for the first time. A 57-year-old Japanese female presented with persistent swelling of the right neck region. Physical examination revealed a tumour in the right parotid gland, and FNA examination of the tumour was performed following surgical resection. The Papanicolaou smear demonstrated the presence of fully necrotic cell clusters of varying sizes in a necrotic background. Only a few viable cell clusters were observed. These cells were polygonal to spindle-shaped, and exhibited large round to oval nuclei with a conspicuous nucleoli. Although nuclear pleomorphism was noted, the nuclear chromatin was fine. No neoplastic myoepithelial cells were observed. Therefore, it was suspected that these cells were malignant. Immunocytochemical analysis indicated that these atypical cells were positive for $\mathrm{p} 40$. Histopathological examination of the resected parotid gland tumour revealed infarcted PA. Our study revealed that the presence of atypical squamous metaplastic cells in a necrotic background was a characteristic cytological feature of infarcted PA, and that these atypical squamous cells can have large nuclei with conspicuous nucleoli and exhibit pleomorphism. Although cytological diagnosis of infarcted PA is difficult, pathologists and cytopathologists must consider the presence of atypical metaplastic squamous cells in benign salivary gland tumours, including infarcted PA.
\end{abstract}

Correspondence to: Dr Mitsuaki Ishida, Department of Pathology and Laboratory Medicine, Kansai Medical University, 2-5-1 Shinmachi, Hirakata, 573-1010 Osaka, Japan

E-mail: ishidamt@hirakata.kmu.ac.jp

Key words: pleomorphic adenoma, infarction, squamous metaplasia, salivary gland

\section{Introduction}

Fine-needle aspiration (FNA) cytology is a well-established, safe and useful diagnostic procedure for salivary gland lesions. Cytological features of pleomorphic adenoma (PA) are well recognized, and its diagnosis using FNA specimens is usually straightforward (1). However, some diagnostic pitfalls and difficulties have been reported for PA, such as prominent squamous metaplasia, predominant cellular component without chondromyxoid stroma, and the presence of nuclear atypia (1). Infarction following the FNA procedure is well-recognized phenomenon in case of some organs including thyroid (2). This phenomenon has also been reported in the salivary gland (3-5), and previous FNA procedure may induce histological alterations including squamous metaplasia, infarction, necrosis, and haemorrhage, resulting in potential overdiagnosis (5). Although extremely rare, it has been documented that spontaneous infarction without previous FNA procedure can occur in salivary gland tumours. To our knowledge, there are very few reports (6-8) and so this is only fourth cytological case report of spontaneous infarction of PA of the parotid gland using immunocytochemical analysis for the first time.

\section{Case report}

A 57-year-old Japanese female presented with persistent swelling of the right neck region. Physical examination revealed a relatively well-circumscribed tumour in the right parotid gland. FNA examination of the right parotid gland tumour was performed.

The Papanicolaou smear of the FNA specimens revealed the presence of variable-sized completely necrotic cell clusters in a necrotic background accompanying neutrophils (Fig. 1A). Only a few viable cell clusters were observed, along with a few non-neoplastic acinar cells (Fig. 1B). These cells were polygonal to spindle-shaped and had large round to oval nuclei with conspicuous nucleoli (Fig. 1B). Although nuclear pleomorphism was noted, the nuclear chromatin was fine and evenly distributed (Fig. 1B). No non-nucleated squamous cells were observed. Moreover, typical neoplastic myoepithelial cells were not observed, and the Giemsa staining did not reveal any chondromyxoid material. Presence of these cytological 

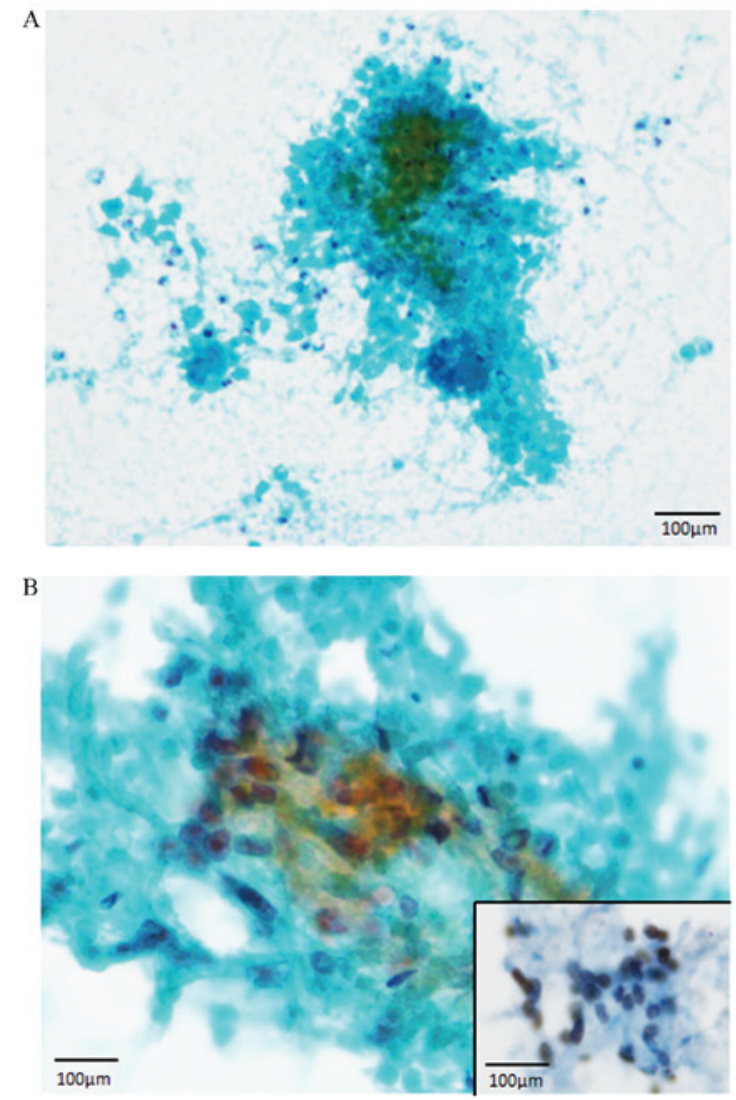

Figure 1. Cytological features of the fine-needle aspiration of the parotid gland tumour. (A) Completely necrotic cell clusters are present in a necrotic background (Papanicolaou stain, x200). (B) Atypical cells are polygonal to spindle-shaped with round to oval nuclei and conspicuous nucleoli. Although nuclear pleomorphism is noted, the nuclear chromatin is fine (Papanicolaou stain, magnification, $\mathrm{x} 400$ ). Immunocytochemical analysis revealing $\mathrm{p} 40$ expression in these atypical cells (inset, magnification, $\mathrm{x} 400)$.

features led to suspicion of malignancy (carcinoma, not specified). Atypical polygonal to spindle-shaped cells showed positive nuclear reactivity for $\mathrm{p} 40$ (BC28, Roche) by immunocytochemical analysis (using archival slides and an autostainer (XT System Benchmark, Roche, Basel, Switzerland) (Fig. 1B, inset).

The surgical resection of the right parotid gland tumour was performed. Histopathological examination of the resected parotid gland tumour revealed a well-circumscribed tumour, measuring $2.5 \times 2.5 \mathrm{~cm}$ in diameter, and a cystic cavity filled with necrotic tissue (Fig. 2A). Atypical squamous metaplastic cells were present around this cavity (Fig. 2B). These cells were polygonal to spindle-shaped, and they exhibited large round to oval nuclei containing a conspicuous nucleoli (Fig. 2C). Although nuclear pleomorphism was noted, no mitotic features were observed in these metaplastic cells. Conventional PA component, composed of proliferating neoplastic myoepithelial cells with bland, round nuclei within a myxochondromatous material and occasional ductal formation was observed around the cavity (Fig. 2D). Accordingly, a diagnosis of PA with spontaneous infarction was made. Post-operative course was uneventful, and no tumor recurrence has been observed during 4 months of medical follow-up.
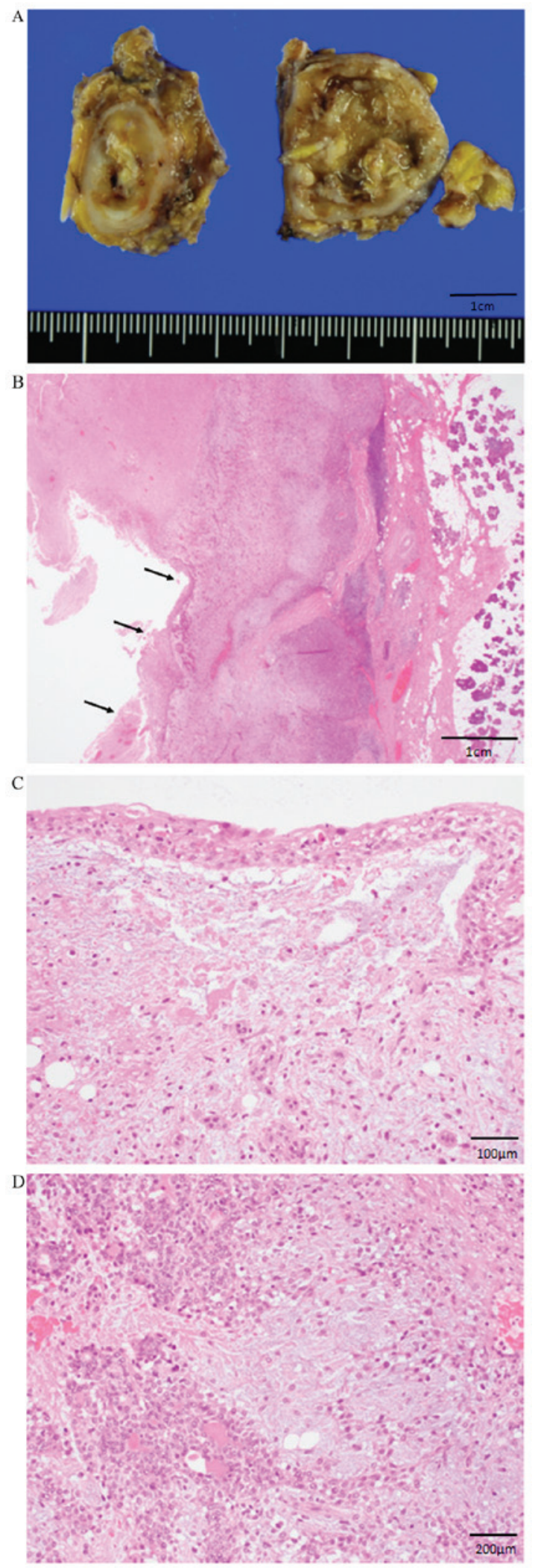

Figure 2. Histopathological features of the parotid gland tumour. (A) Macroscopic examination showing necrotic material filled in the centre of the tumour. (B) Well-circumscribed tumour with cystic cavitation filled with necrotic material in the centre. Cystic cavity is covered with atypical squamous cells (arrows), and the conventional pleomorphic adenoma component is noted at the periphery (haematoxylin and eosin, magnification, $\mathrm{x} 20$ ). (C) Atypical squamous cells exhibit large, round to oval nuclei and nuclear pleomorphism (haematoxylin and eosin, magnification, $\mathrm{x} 400$ ). (D) Conventional pleomorphic adenoma component. Proliferation of neoplastic myoepithelial cells within myxoid material (haematoxylin and eosin, magnification, $\mathrm{x} 200$ ). 


\section{Discussion}

Here, we describe the first cytological report of infarcted PA with immunocytochemical analysis of p40. Infarction following FNA procedure in PA is a well-known phenomenon; however, it might be associated with potential diagnostic challenges (5). According to a study that evaluated the histological alterations of parotid gland lesions associated with pre-operative FNA, the resected specimens exhibited acute and chronic haemorrhage and inflammation (100\%); squamous metaplasia ( $80 \%)$, which is considered as a reparative process secondary to infarction; infarction and necrosis (40\%); and stromal hyalinization (30\%) (5). Among these characteristics, the presence of squamous metaplasia and necrosis could lead to a histological misdiagnosis of carcinoma, such as squamous cell carcinoma and mucoepidermoid carcinoma (5).

Albeit extremely rare, infarction can occur in PA without previous FNA procedure. Layfield et al described the first cytological case of spontaneous infarcted PA (8). Since then, only three additional cytological cases, including the present one, have been reported in the English-language literature $(6,7)$. The cytological features of the infarcted PA are follows: i) Presence of atypical squamous metaplastic cells in a necrotic background (4/4 cases), ii) presence of anucleated squamous cells ( $3 / 4$ cases), and iii) presence of sheets of oncocytic cells without atypia (1/4 case) (6-8). In all of these cases, an initial cytological diagnosis of probable malignancy was made (6-8). Atypical squamous metaplastic cells in a necrotic background is a common cytological feature of infarcted PA; these cells could show extremely atypical features, including ovoid to elongated large nuclei, conspicuous nucleoli with irregular shapes and sizes, and marked pleomorphism (6). These atypical cells were considered as metaplastic squamous cells, as per the histopathological features of the resected tumour. However, immunocytochemical confirmation has not been performed. Our immunocytochemical analysis clearly showed nuclear p40 expression in the atypical cells with large nuclei containing conspicuous nucleoli and nuclear pleomorphism. Accordingly, these atypical cells in the cytological specimens of the infarcted PA were confirmed as metaplastic squamous cells.

Cytological differential diagnostic considerations in cases where atypical squamous cells are present in a necrotic background include Warthin's tumour and mucoepidermoid carcinoma. Presence of necrotic material is a common feature of Warthin's tumour, and squamous cells are occasionally noted $(9,10)$. The cytological specimens of Warthin's tumour usually contain characteristic oncocytic cells, along with squamous cells (10) which is a useful in diagnosis of Warthin's tumour. Mucoepidermoid carcinoma has at least some mucous and/or intermediate cells (10). These features may aid differential diagnosis from infarcted PA.

In conclusion, the present report describes the cytological features of infarcted PA through immunocytochemical analysis of p40 expression. Presence of atypical metaplastic squamous cells positive for p40 in a necrotic background was characteristic cytological feature of infarcted PA. Although a necrotic background is an important indication of malignancy in the cytological diagnosis of salivary gland tumours (11), the presence of atypical metaplastic squamous cells in a necrotic background does not directly indicate malignant diagnosis. Though cytological diagnosis of infarcted PA is extremely difficult, pathologists and cytopathologists must consider that atypical metaplastic squamous cells may be present in benign salivary gland tumours, including infarcted PA.

\section{Acknowledgements}

Not applicable.

\section{Funding}

No funding was received.

\section{Availability of data and materials}

All data generated or analyzed during this study are included in this published article.

\section{Authors' contributions}

$\mathrm{HI}$ and MI conceived of and designed the study. HI, MI, KO, $\mathrm{KS}, \mathrm{YE}, \mathrm{SY}, \mathrm{TF}, \mathrm{HI}$ and KT collected and analyzed the data. $\mathrm{HI}$ and MI drafted the manuscript and figures. All authors read and approved the final manuscript.

\section{Ethics approval and consent to participate}

This study was conducted in accordance with the Declaration of Helsinki and the study protocol was approved by the Institutional Review Board of Kansai Medical University Hospital (Approval no. 160954). Informed consent was obtained.

\section{Patient consent for publication}

Opt-out consent was obtained from participant of the current study.

\section{Competing interests}

The authors declare that they have no competing interests.

\section{References}

1. Klijanienko $\mathrm{J}$ and Vielh P: Fine-needle sampling of salivary gland lesions. I. Cytology and histology correlation of 412 cases of pleomorphic adenoma. Diagn Cytopathol 14: 195-200, 1996.

2. Kini SR: Post-fine-needle biopsy infarction of thyroid neoplasms: A review of 28 cases. Diagn Cytopathol 15: 211-220, 1996.

3. Pabuççuoglu HU, Lebe B, Sarioglu S and Lebe E: Infarction of pleomorphic adenoma: A rare complication of fine-needle aspiration obscuring definitive diagnosis. Diagn Cytopathol 24: 301-303, 2001.

4. Pinto RG, Couto F and Mandreker S: Infarction after fine needle aspiration. A report of four cases. Acta Cytol 40: 739-741, 1996.

5. Li S, Baloch ZW, Tomaszewski JE and LiVolsi VA: Worrisome histologic alterations following fine-needle aspiration of benign parotid lesions. Arch Pathol Lab Med 124: 87-91, 2000.

6. Fulciniti F, Losito NS, Botti G, Manola M and Ionna F: Spontaneous infarction of pleomorphic adenoma: Report of a case simulating malignancy on fine-needle cytology sample. Diagn Cytopathol 38: 430-434, 2010. 
7. Behzatoglu K, Bahadir B, Huq GE and Kaplan $\mathrm{HH}$ Spontaneous infarction of a pleomorphic adenoma in parotid gland: Diagnostic problems and review. Diagn Cytopathol 32: 367-369, 2005.

8. Layfield LJ, Reznicek M, Lowe M and Bottles K: Spontaneous infarction of a parotid gland pleomorphic adenoma. Report of a case with cytologic and radiographic overlap with a primary salivary gland malignancy. Acta Cytol 36: 381-386, 1992.

9. Cobb CJ, Greaves TS and Raza AS: Fine needle aspiration cytology and diagnostic pitfalls in Warthin's tumor with necrotizing granulomatous inflammation and facial nerve paralysis: A case report. Acta Cytol 53: 431-434, 2009.
10. Klijanienko J and Vielh P: Fine-needle sampling of salivary gland lesions. II. Cytology and histology correlation of 71 cases of Warthin's tumor (adenolymphoma). Diagn Cytopathol 16: 221-225, 1997.

11. Okano K, Ishida M, Sandoh K, Fujisawa T, Iwai H and Tsuta K: Cytological features of carcinoma ex pleomorphic adenoma of the salivary glands: A diagnostic challenge. Diagn Cytopathol 48: 149-153, 2020.

This work is licensed under a Creative Commons Attribution-NonCommercial-NoDerivatives 4.0 International (CC BY-NC-ND 4.0) License. 